ISSN 0103-9954

\title{
ESTRUTURA FITOSSOCIOLÓGICA DO COMPONENTE ARBÓREO DE UM FRAGMENTO DE FLORESTA OMBRÓFILA DENSA NA MATA SUL DE PERNAMBUCO, NORDESTE DO BRASIL
}

\author{
PHYTOSOCIOLOGICAL STRUCTURE OF THE ARBOREOUS COMPONENT OF DENSE \\ OMBROPHYLOUS FOREST FRAGMENT IN SOUTH BUSH OF PERNAMBUCO STATE, \\ NORTHEASTERN BRAZIL
}

\author{
Roberto Felix Costa Junior ${ }^{1}$ Rinaldo Luiz Caraciolo Ferreira ${ }^{2}$ Maria Jesus Nogueira Rodal ${ }^{3}$ \\ Ana Lícia Patriota Feliciano ${ }^{4}$ Luiz Carlos Marangon ${ }^{5}$ Wegliane Campelo da Silva ${ }^{6}$

\section{RESUMO}

O objetivo deste trabalho foi descrever a fisionomia e a estrutura de um fragmento de Floresta Ombrófila Densa localizado no município de Catende, Pernambuco. Foram mensuradas todas as árvores com de DAP (diâmetro a altura do peito) $\geq 4,77 \mathrm{~cm}$ em quarenta parcelas $(10 \times 25 \mathrm{~m})$, instaladas sistematicamente em cinco transectos. Observou-se 1.049 indivíduos, distribuídos em 91 espécies, 64 gêneros e 37 famílias botânicas. Mimosaceae e Lauraceae foram as famílias com maior número de espécies enquanto que Anacardiaceae e Moraceae tiveram o maior número de indivíduos. O índice do Shannon $\left(\mathrm{H}^{\prime}\right)$ foi de 3,83 nats/ind. A área basal total, a altura e o diâmetro (médio e máximo) foram $23,59 \mathrm{~m}^{2} / \mathrm{ha}, 13,57 \mathrm{~m}( \pm 7,13)$, $45 \mathrm{~m}, 13,20 \mathrm{~cm}( \pm 10,60)$ e 127,32 cm respectivamente. As dez espécies de maior valor de importância foram Tapirira guianensis Aubl., Dialium guianense (Aubl.) Sandwith, Helicostylis tomentosa (Poepp. \& Endl.) Rusby, Thyrsodium spruceanum Benth., Plathymenia foliolosa Benth., Brosimum discolor Schott, Parkia pendula (Willd.) Benth. ex Walp., Schefflera morototoni (Aubl.) Maguire, Steyerm. \& Frodin, Eschweilera ovata (Cambess.) Miers e Cecropia palmata Willd. A distribuição diamétrica apresentou curva semelhante à forma de J-invertido, prevista para uma floresta ineqüiânea em estágio de sucessão secundária.

Palavras-chave: distribuição diamétrica; distribuição hipsométrica; florística.

\section{ABSTRACT}

This work aimed to describe physiognomy and structure of a Dense Ombrophyllous Forest fragment located in Catende, Pernambuco. All trees with DAP (breast height diameter) $\geq 4.77 \mathrm{~cm}$ were measured in 40 $10 \times 25 \mathrm{~m}$ plots, systematically installed in five transects. A total of 1049 individuals were observed, distributed in 91 species, 64 genera and 37 botanical families. Mimosaceae and Lauraceae were the families with higher species number, while Anacardiaceae and Moraceae had the highest individuals numbers. Shannon index (H') was 3.83 nats/ind. Total basal area, height and diameter (average and maximum) were $23.59 \mathrm{~m}^{2} / \mathrm{ha}, 13.57 \mathrm{~m}( \pm 7.13), 45 \mathrm{~m}, 13.20 \mathrm{~cm}( \pm 10.60)$ and $127.32 \mathrm{~cm}$, respectively. The species with higher importance values were Tapirira guianensis Aubl., Dialium guianense (Aubl.) Sandwith, Helicostylis tomentosa (Poepp. \& Endl.) Rusby, Thyrsodium spruceanum Benth., Plathymenia foliolosa Benth., Brosimum discolor Schott, Parkia pendula (Willd.) Benth. ex Walp., Schefflera morototoni (Aubl.) Maguire, Steyerm. \& Frodin, Eschweilera ovata (Cambess.) Miers and Cecropia palmata Willd. Diametric

1. Engenheiro Florestal, MSc., Programa de Pós-Graduação em Ciências Florestais, Universidade Federal de Pernambuco, Rua Dr. Orlando Cabral, 27, Bairro Centro, CEP 54080-300, Jaboatão dos Guararapes (PE). rfflorestal@yahoo.com.br

2. Engenheiro Florestal, Dr., Professor do Departamento de Ciências Florestais, Universidade Federal de Pernambuco, Rua Dom Manoel de Medeiros, s/n, Bairro Dois Irmãos, CEP 52171-900, Recife (PE). Bolsista do CNPq. rinaldo@dcfl.ufrpe.br

3. Bióloga, Dr., Professora do Departamento de Biologia, Universidade Federal de Pernambuco, Rua Dom Manoel de Medeiros, s/n, Bairro Dois Irmãos, CEP 52171-900, Recife (PE). Bolsista do CNPq. mrodal@terra.com.br

4. Engenheira Florestal, Dr ${ }^{\mathrm{a}}$, Professora do Departamento de Ciências Florestais, Universidade Federal de Pernambuco, Rua Dom Manoel de Medeiros, s/n, Bairro Dois Irmãos, CEP 52171-900, Recife (PE).licia@dcfl.ufrpe.br

5. Engenheira Florestal, Dra ${ }^{\mathrm{a}}$, Professora do Departamento de Ciências Florestais, Universidade Federal de Pernambuco, Rua Dom Manoel de Medeiros, s/n, Bairro Dois Irmãos, CEP 52171-900, Recife (PE). marangon@dcfl.ufrpe

6. Engenheiro Florestal, MSc., Programa de Pós-Graduação em Ciências Florestais, Universidade Federal de Pernambuco, Rua Dom Manoel de Medeiros, s/n, Bairro Dois Irmãos, CEP 52171-900, Recife (PE). wellcampelo@yahoo.com

Recebido para publicação em 26/06/2006 e aceito em 22/10/2007. 
distribution had an inverted-J-like curve, predicted for a forest unequal in ages in a secondary succession stage.

Keywords: diametrical distribution; hypsometric distribution; floristics.

\section{INTRODUÇÃO}

A Floresta Tropical representa uma organização biológica de grande importância do ponto de vista da biodiversidade, nela se encontram diferentes formações vegetacionais que servem de abrigo a diversas formas de vida e que, no decorrer do tempo, tem servido a atividades desordenadas e outras práticas de exploração. Com a destruição acelerada dessas florestas, grande parte da biodiversidade presente nesses ecossistemas está se perdendo, antes mesmo que se tenha inteiro conhecimento de sua riqueza natural (BORÉM e OLIVEIRA-FILHO, 2002).

Dentre as formações florestais tropicais que ocorrem no Brasil, pode-se destacar a Floresta Ombrófila Densa, que se encontra distribuída ao longo de toda sua costa litorânea, sendo definida por Vanini e Rodrigues (2003) como resultante de díspares combinações de espécies em cada parte ou unidade da floresta, formando um grande mosaico constituído por manchas de várias idades e diferentes estágios sucessionais, originadas por perturbações externas e processos de sucessão secundária.

A grande diversidade florística e o alto índice de endemismo da Floresta Ombrófila Densa são fatores de grande importância e requerem o desenvolvimento de estudos florísticos e fitossociológicos para o entendimento dos diferentes ecossistemas florestais da região tropical. (LEITÃO FILHO, 1987; JOLY et al.; 1991; GENTRY, 1995).

De acordo com Oliveira et al. (2001), o estudo fitossociológico é uma maneira adequada de buscar respostas iniciais da organização da vegetação e tem se revelado uma análise importante na caracterização da comunidade vegetal. Segundo Borém e Ramos (2001), o conhecimento da composição florística e da estrutura fitossociológica das espécies tem muito a contribuir para a conservação, recuperação e o manejo desses ecossistemas.

Sendo assim, para a conservação das formações florestais, é importante antes de qualquer plano de ação, conhecer a composição e estrutura dos remanescentes, o que é de suma relevância para que se façam intervenções e elaboração de planos de manejo sustentável.

No município de Catende, PE, estão localizados remanescentes de Floresta Ombrófila Densa, que também vem tendo sua biodiversidade ameaçada pela exploração dos recursos, considerando a substituição da vegetação por plantações agrícolas, sobretudo de cana-de-açúcar, pois Catende é conhecida historicamente pela sua grande produção açucareira, que foi e ainda é fonte de renda para a maioria de seus habitantes.

O objetivo deste trabalho foi realizar um estudo fitossociológico do componente arbóreo, com o intuito de caracterizar a estrutura de um fragmento de Floresta Ombrófila Densa, localizado no município de Catende, PE.

\section{MATERIAIS E MÉTODOS}

\section{Área de estudo}

O remanescente de Floresta Ombrófila Densa estudado é conhecido localmente por Mata das Caldeiras, possui 38,56 ha e está localizado no município de Catende, PE, distando aproximadamente $11 \mathrm{Km}$ da sua sede municipal, nas coordenadas $8^{\circ} 63^{\prime}$ '59' 'S e $35^{\circ} 77^{\prime}$ '74', a uma altitude de $327 \mathrm{~m}$ (Figura 1). 


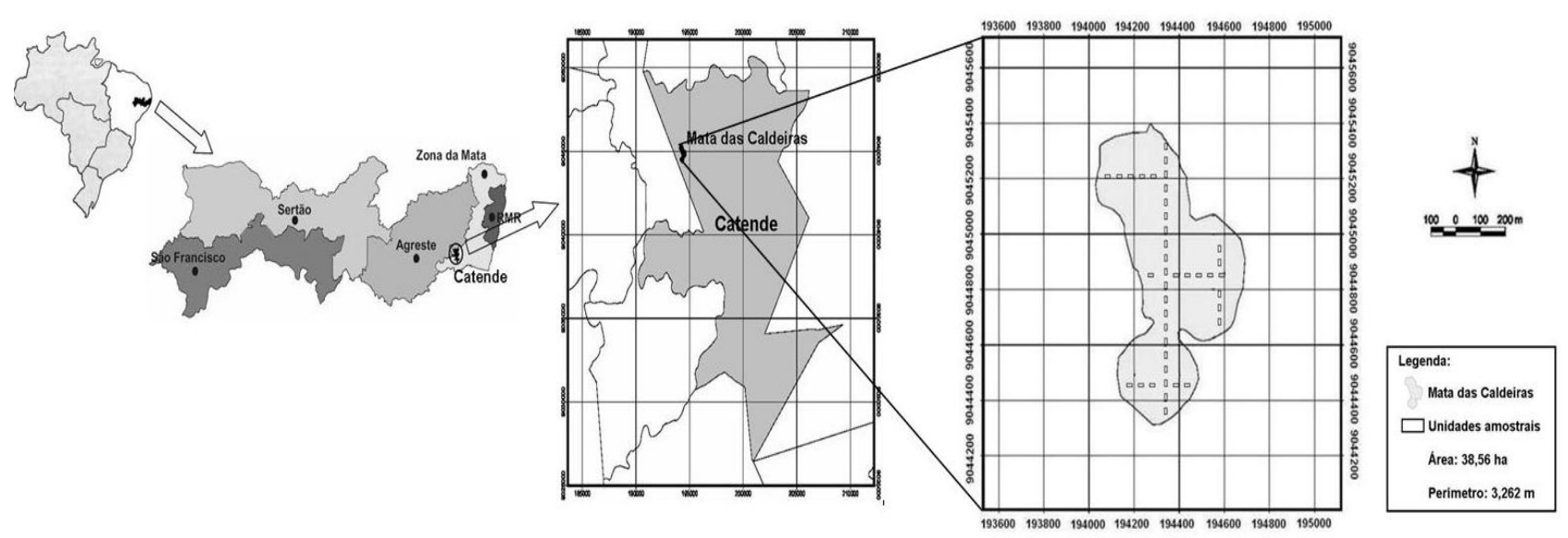

FIGURA 1: Localização da área de estudo e dos transectos instalados no fragmento Mata das Caldeiras, Catende, Pernambuco, Brasil.

FIGURE 1: Study area and transect location in Mata das Caldeiras fragment, Catende, Pernambuco, Brazil.

O município de Catende está situado na mesorregião da Mata Pernambucana, mais precisamente na microrregião da mata úmida. A geologia é caracterizada, basicamente, pelo Complexo Magmático Granitóide e o solo predominantemente é do tipo Latossolo vermelho amarelo distrófico. O relevo predominante varia de ondulado a forte ondulado. No tocante à sua hidrografia, o município é drenado pelo rio Una e pelo rio Pirangi. O clima é do tipo As' segundo a classificação de Köppen com temperatura média anual superior $22^{\circ} \mathrm{C}$ e precipitação média anual de $1.414 \mathrm{~mm}$. (CONDEPE, 1987).

\section{Métodos}

Foram instaladas quarenta0 parcelas de $250 \mathrm{~m}^{2}(10 \times 25 \mathrm{~m})$, alocadas sistematicamente, em cinco transectos (Figura 1). Dentro dos transectos as parcelas distaram $25 \mathrm{~m}$. Foram amostrados apenas os indivíduos arbóreos vivos com DAP (diâmetro a 1,30m do solo) $\geq 4,77 \mathrm{~cm}$, que foram etiquetados e tiveram o DAP, a altura total e suas características dendrológicas anotadas além da coleta do material botânico, preferencialmente fértil.

\section{Análise dos dados}

O material coletado foi identificado mediante bibliografia especializada, por meio de comparação com material de herbário e por especialistas. As espécies foram organizadas por família seguindo o sistema de classificação de Cronquist (1988).

A suficiência das espécies amostradas foi otimizada utilizando-se o procedimento REGRELRP do programa SAEG - Sistema para Análise Estatística e Genética (UFV, 1997) conforme adotado por Ferreira e Vale (1992). Esse procedimento é apropriado para análise de regressão de modelos descontínuos, compostos de uma parte linear crescente e de uma na forma de plateau (UFV, 1997).

A análise da estrutura horizontal foi realizada partindo da estimativa dos seguintes parâmetros fitossociológicos: densidade, freqüência, dominância, valor de importância (MUELLER-DOMBOIS e ELLENBERG, 1974). A diversidade foi analisada por meio do índice de Shannon (MAGURRAN, 1988). A análise da distribuição diamétrica foi elaborada por meio de histograma (intervalo de $5 \mathrm{~cm}$ ) com o número de indivíduos por classes de diâmetro, em que o valor da primeira classe foi $4,77 \mathrm{~cm}$, que corresponde ao valor mínimo de diâmetro adotado como critério de inclusão no levantamento, e última classe com o intervalo aberto a direita, contemplou os indivíduos com DAP $\geq 104,77 \mathrm{~cm}$.

A caracterização da ocupação do espaço vertical foi realizada por meio de histograma (intervalo de 5 m) com o número de indivíduos, classe de altura e área basal.

\section{RESULTADOS E DISCUSSÃO}

$\mathrm{Na}$ análise da suficiência amostral, pôde-se verificar que ocorreu a formação do plateau partindo da área igual a $5.500 \mathrm{~m}^{2}$, a qual representa a área mínima necessária para a caracterização da composição 
florística do fragmento estudado dando como satisfatória a amostragem realizada (Figura 2).

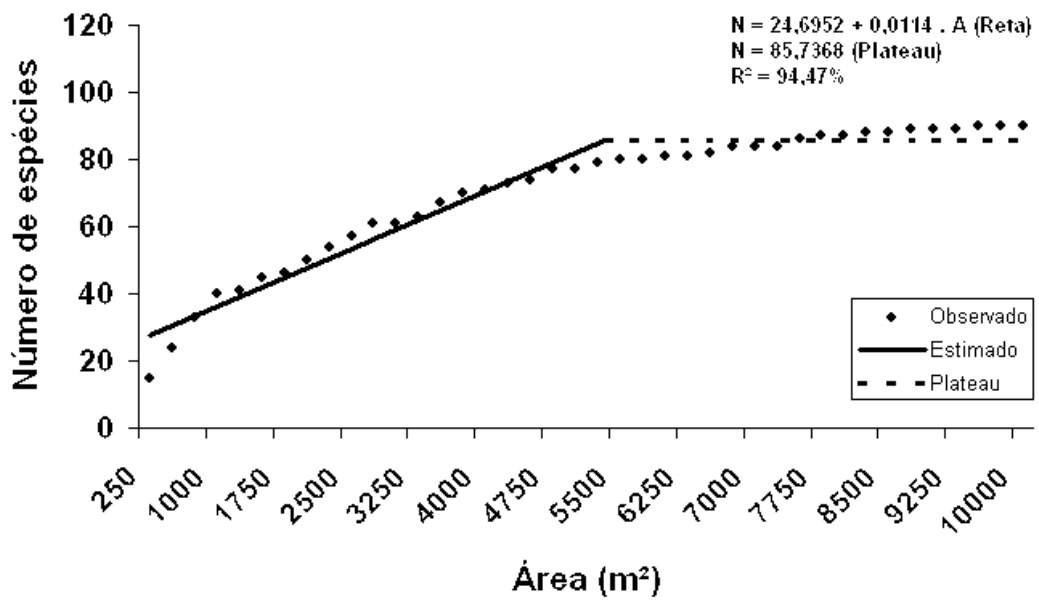

FIGURA 2: Suficiência amostral do levantamento realizado no fragmento Mata das Caldeiras, Catende, PE. FIGURE 2: Sampling sufficiency for the arboreal plants in "Mata das Caldeiras", Catende, Pernambuco.

Foram amostrados 1.049 indivíduos correspondendo a uma área basal total de $23,59 \mathrm{~m}^{2} / \mathrm{ha}$. Nas Figuras 3 e 4, verifica-se que o maior número de indivíduos ocorreu na classe de 5,1 a $10 \mathrm{~m}(36,89 \%)$. Todavia, os indivíduos dessa classe de altura contribuíram com $9,45 \%$ da área basal total. Apesar da classe de 15,1 a 20 m apresentar menor número de indivíduos (18,39\%) foi a de maior área basal, o que sugere que o dossel da floresta esteja nesse intervalo.

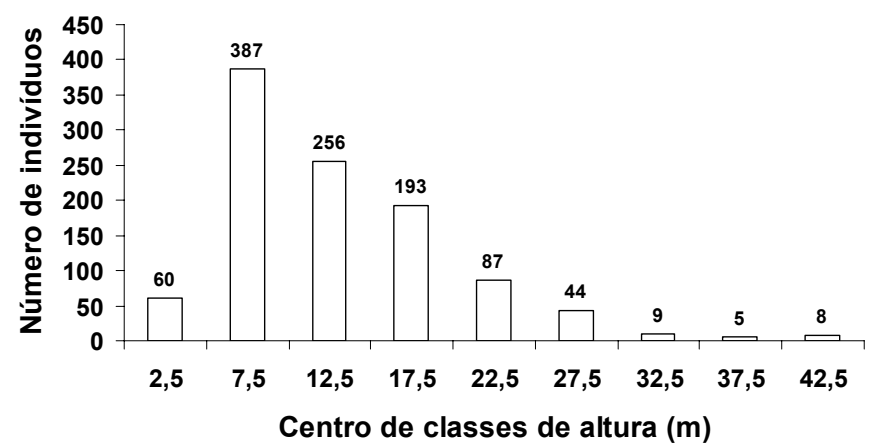

FIGURA 3: Distribuição do número de indivíduos arbóreos por classe de altura amostrados na Mata das Caldeiras, Catende, PE.

FIGURE 3: Arboreal plants distribution by height class, "Mata das Caldeiras" fragment, Catende, Pernambuco.

Com relação à área basal, o valor obtido $\left(23,59 \mathrm{~m}^{2} / \mathrm{ha}\right)$ na Mata das Caldeiras foi próximo ao encontrado por Lins-e-Silva e Rodal (prelo) que foi de $24,7 \mathrm{~m}^{2} / \mathrm{ha}$, porém foi menor aos encontrados por Guedes (1998), Siqueira et al. (2001) e Silva Júnior (2004) que constataram 44,7, 27,5 e 32,58 $\mathrm{m}^{2} / \mathrm{ha}$ respectivamente.

Analisando os valores de área basal total entre as classes de altura, observou-se que a maior concentração de área basal total ocorreu nas classes entre 15,1 e $30 \mathrm{~m}$ com $62,65 \%$; enquanto que os indivíduos com até 15 metros representaram $25,73 \%$ e os indivíduos maiores de $30 \mathrm{~m}$ por apenas $11,61 \%$ (Figura 4). 


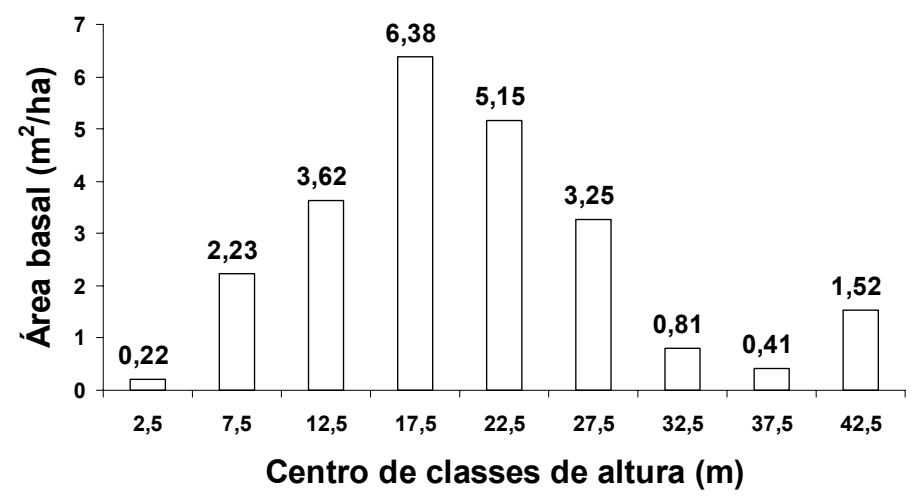

FIGURA 4: Distribuição da área basal por classe de altura dos indivíduos arbóreos amostrados na Mata das Caldeiras, Catende, PE.

FIGURE 4: Basal area distribution by height class of arboreal plants of "Mata das Caldeiras", Catende, Pernambuco.

Os resultados obtidos na análise da ocupação do espaço vertical indicam uma possível estratificação formada pelo estrato inferior, menor que $15 \mathrm{~m}$, pelo estrato médio cujos indivíduos atingem entre 15,1 e 30,0 $\mathrm{m}$ e o estrato superior com indivíduos com altura maior que $30 \mathrm{~m}$.

Pode-se inferir também que essa estratificação encontrada no fragmento estudado apresenta comportamento de floresta ineqüiânea secundária em estágios iniciais de sucessão.

O valor médio estimado do DAP foi de $13,20 \mathrm{~cm}$, tendo como o máximo $127,32 \mathrm{~cm}$ e o mínimo $4,77 \mathrm{~cm}$. A primeira classe $(4,77$ a $9,77 \mathrm{~cm})$ foi a que obteve o maior número de indivíduos $(531) \mathrm{com}$ $50,61 \%$, decrescendo na segunda classe em quase a metade.

A curva de distribuição de diâmetros dos indivíduos (Figura 5) segue o padrão característico de florestas ineqüiâneas, ou seja, apresenta uma distribuição exponencial na forma de J-invertido (Assmann, 1970), em que a maior freqüência de indivíduos se encontra nas classes de diâmetros menores.

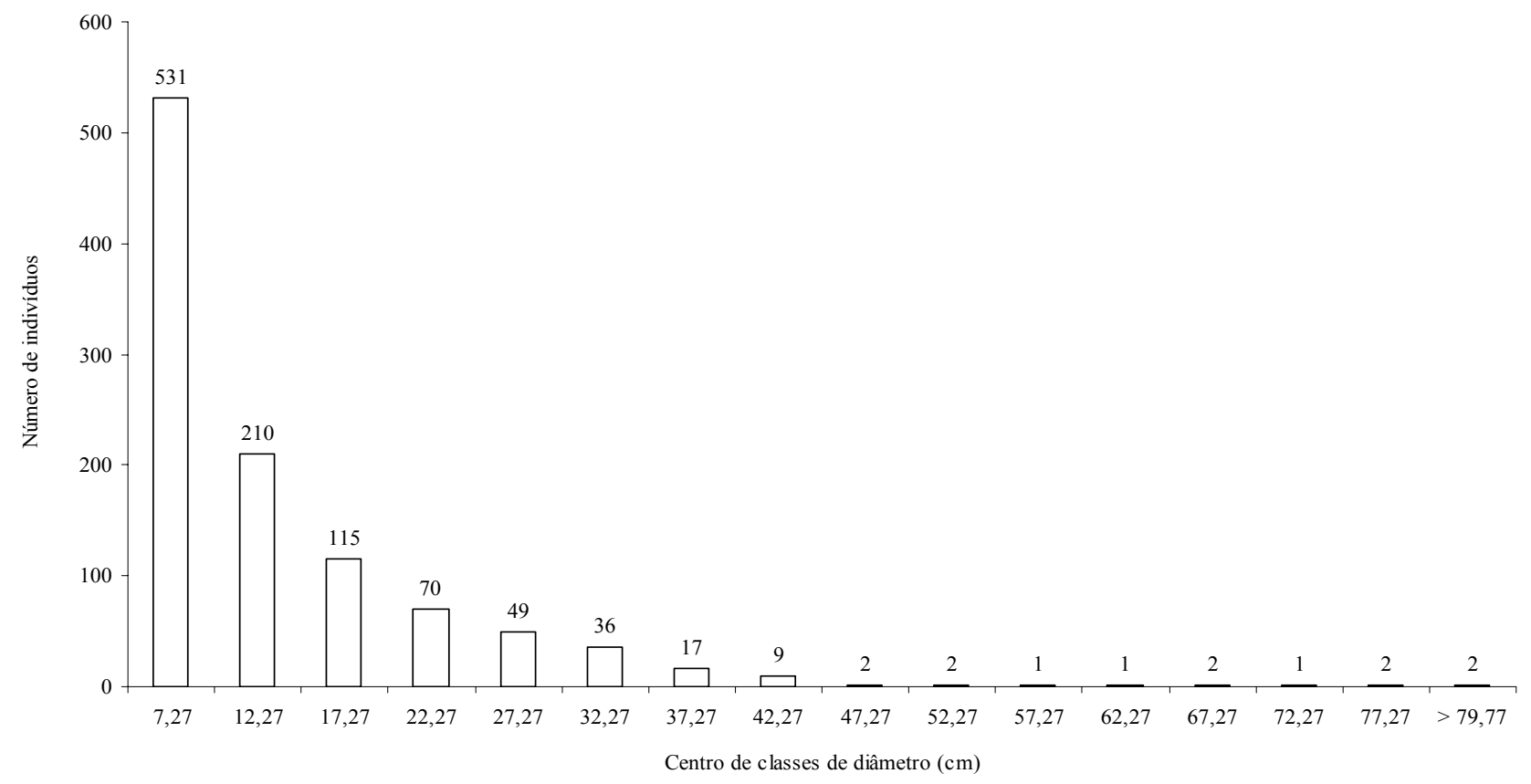

FIGURA 5: Distribuição diamétrica dos indivíduos arbóreos amostrados na Mata das Caldeiras, Catende, PE. FIGURE 5: Diameter distribution of arboreal plants of Mata das Caldeiras, Catende, Pernambuco.

Meyer (1952) e Assmann (1970) constataram que tal distribuição formando uma curva semelhante à 
forma de J invertido é prevista para formações florestais ineqüiânea. Silva Júnior e Silva (1988) relataram que além de ineqüiânea, essas formações são classificadas como secundária em estágios iniciais de sucessão. O que corrobora com a afirmação de Machado et al. (2004) que a quase totalidade dos inventários de comunidades arbóreo-arbustivas de florestas naturais apresenta uma distribuição diamétrica seguindo o modelo $\mathrm{J}$ invertido ou exponencial negativo.

Segundo Martins (1991), a maior densidade de indivíduos menores não indica ausência de problemas de regeneração, mencionando a necessidade de uma análise mais detalhada, em nível específico e com um grupo maior de espécies para permitir interpretações mais seguras das distribuições diamétricas.

Para uma conclusão mais contundente sobre o comportamento da distribuição diamétrica dessas espécies, faz-se necessário um estudo de regeneração natural e da ecologia, atrelado a um estudo etnobotânico para verificar prováveis níveis de exploração dessas espécies pela comunidade local.

Os 1.049 indivíduos amostrados pertencem a 38 famílias botânicas, 64 gêneros e noventa espécies. Dessas espécies, sete foram identificadas somente em nível de gênero e apenas um táxon permaneceu indeterminado.

Em termos de riqueza de espécies, as dez famílias mais bem representadas na área em estudo foram: Mimosaceae (10), Lauraceae (6), Annonaceae (5), Moraceae (5), Anacardiaceae (4), Flacourtiaceae (4), Meliaceae (4), Sapindaceae (4), Sapotaceae (4) e Caesalpiniaceae (3). Essas famílias representaram 54,44\% da flora amostrada, podendo-se ainda observar que 18 famílias (47,37\% do total) apresentaram uma única espécie.

Comparando com estudos em Floresta Ombrófila Densa de Terras Baixas de Pernambuco, notou-se que a família Mimosaceae também foi a que mais se destacou entre às outras identificadas (GUEDES, 1998; SIQUEIRA et al., 2001; SILVA, 2004; SILVA JÚNIOR, 2004; FEITOSA, 2004; LINS-E-SILVA e RODAL, prelo).

As dez espécies que apresentaram maior valor de importância (VI) da área estudada, em ordem decrescente, foram: Tapirira guianensis, Dialium guianense, Helicostylis tomentosa, Thyrsodium spruceanum, Plathymenia foliolosa, Brosimum discolor, Parkia pendula, Schefflera morototoni, Eschweilera ovata e Cecropia palmata (Tabela 1).

A espécie de maior VI da Mata das Caldeiras foi a Tapirira guianensis a qual se destacou por ser a detentora dos maiores valores de densidade, freqüência e dominância. Essa espécie também foi apontada como uma das principais em termos de maiores valores de importância por Guedes (1998); Siqueira et al. (2001), Feitosa (2004), Lins-e-Silva e Rodal (prelo). O que corrobora com Guedes (1998) ao relatar que a elevada densidade de indivíduos dessa espécie indica o processo de antropização do fragmento passou, considerando que a espécie é encontrada em diversos estágios de desenvolvimento.

Dentre as espécies de maiores VI destacam-se também Plathymenia foliolosa e Parkia pendula pelos seus altos valores de dominância, motivo pelo qual alcançaram o quinto e sétimo lugares conseqüentemente, pois seus valores de dominância relativa contribuíram em 65,04 e 70,07\% para o seu valor de VI. As espécies Dialium guianense, Helicostylis tomentosa e Thyrsodium spruceanum também se destacaram por terem sido as espécies amostradas com valores expressivos de densidade e por último a Eschweilera ovata pela elevada freqüência (Tabela 1).

As dez espécies mais representativas em termos de densidade, ordenadas de forma decrescente do maior valor, foram: Tapirira guianensis, Helicostylis tomentosa, Thyrsodium spruceanum, Dialium guianense, Brosimum discolor, Cecropia palmata, Eschweilera ovata, Schefflera morototoni, Plathymenia foliolosa e Pouteria grandiflora (Tabela 1).

Ao comparar a outros estudos em Floresta Ombrófila Densa de Terras Baixas em Pernambuco, a Helicostylis tomentosa também se destacou entre as de maior densidade no estudo de Guedes (1998) e Lins-e-Silva e Rodal (prelo) e a Eschweilera ovata nos de Guedes (1998); Siqueira et al. (2001); Silva Junior (2004) e Feitosa (2004).

As espécies de maior freqüência na Mata das Caldeiras, ordenadas de forma decrescente, foram: Tapirira guianensis, Helicostylis tomentosa, Thyrsodium spruceanum, Dialium guianense, Brosimum 
discolor, Eschweilera ovata, Nectandra cuspidata, Cupania racemosa, Plathymenia foliolosa e Erythroxylum squamatum (Tabela 1). Torna-se importante salientar que a Tapirira guianensis esteve entre as dez espécies mais freqüentes nos trabalhos realizados em Floresta Ombrófila Densa de Terras Baixas de Pernambuco (GUEDES, 1998; SIQUEIRA et al., 2001; FEITOSA, 2004; LINS-E-SILVA e RODAL, prelo) e a Eschweilera ovata (GUEDES, 1998; SIQUEIRA et al., 2001; SILVA JÚNIOR, 2004 e FEITOSA, 2004).

As dez espécies que apresentaram maior dominância da área estudada, em ordem decrescente, foram: Tapirira guianensis, Plathymenia foliolosa, Parkia pendula, Dialium guianense, Quiina sp, Brosimum discolor, Helicostylis tomentosa, Schefflera morototoni, Thyrsodium spruceanum e Cecropia palmata (Tabela 1). A espécie Tapirira guianensis também foi encontrada como detentora de maior dominância em todos os estudos em Floresta Ombrófila Densa de Terras Baixas de Pernambuco (Guedes, 1998; Lins-e-Silva e Rodal, prelo; Siqueira et al., 2001; Silva Júnior, 2004 e Feitosa, 2004).

Considerando os estratos da floresta, as espécies que mais se destacaram em densidade, freqüência e dominância foram: Helicostylis tomentosa, Thyrsodium spruceanum, Dialium guianense, Tapirira guianensis, Brosimum discolor, Eschweilera ovata e Pouteria grandiflora no sub-bosque; Tapirira guianensis, Dialium guianense, Thyrsodium spruceanum, Plathymenia foliolosa, Brosimum discolor, Helicostylis tomentosa e Schefflera morototoni no dossel e Tapirira guianensis, Brosimum discolor e Licania rígida entre as emergentes.

É importante enfatizar também que as espécies Helicostylis tomentosa, Tapirira guianensis, Dialium guianense, Parkia pendula, Brosimum discolor, se destacam entre as demais, em razão da sua representatividade nos diferentes estratos da floresta, estando entre as de maior densidade no sub-bosque, dossel e entre as emergentes.

O índice de diversidade de Shannon (H') da Mata das Caldeiras foi estimado em 3,83 nats/ind., mostrando-se superior à maioria dos estudos realizados em Florestas Ombrofilas Densa de Terras Baixas em Pernambuco de mesmo critério de inclusão (CAP $\geq 15 \mathrm{~cm}$ ) e área amostral (1 ha), citando-se Feitosa (2004) na Mata de Tejipio (2,88 nats/ind,), e Siqueira et al. (2001) na Mata do Zumbi (3,47 nats/ind.) e Silva Júnior (2004) na RESEC Gurjaú (3,91 nats/ind.)

TABELA 1: Parâmetros fitossociológicos das espécies amostradas em um hectare de Floresta Ombrófila Densa na Mata das Caldeiras, Catende, PE. Os valores estão ordem decrescente do valor de importância (VI).

TABLE 1: Phytosociologic parameters of species sampled in one hectare in Dense Ombrophylous Forest, Mata das Caldeiras, Catende, PE. Values ranked by importance value (VI).

\begin{tabular}{|c|c|c|c|c|c|c|c|}
\hline Nome científico & $\begin{array}{r}\text { DoA } \\
\left(\mathrm{m}^{2}\right)\end{array}$ & $\begin{array}{c}\text { DoR } \\
(\%)\end{array}$ & $\begin{array}{c}\text { DA } \\
\text { Ind./ha) }\end{array}$ & $\begin{array}{l}\mathrm{DR} \\
(\%)\end{array}$ & $\begin{array}{l}\text { FA } \\
(\%)\end{array}$ & $\begin{array}{l}\text { FR } \\
(\%)\end{array}$ & VI \\
\hline$\overline{\text { Tapirira guianensis Aubl. }}$ & 3,402 & 14,42 & 97 & 9,25 & 90,0 & 6,00 & 29,67 \\
\hline Dialium guianense (Aubl.) Sandwith & 1,459 & 6,19 & 68 & 6,48 & 75,0 & 5,00 & 17,67 \\
\hline Helicostylis tomentosa (Poepp. \& Endl.) Rusby & 0,955 & 4,05 & 78 & 7,44 & 82,5 & 5,50 & 16,98 \\
\hline Thyrsodium spruceanum Benth. & 0,768 & 3,25 & 77 & 7,34 & 80,0 & 5,33 & 15,93 \\
\hline Plathymenia foliolosa Benth. & 2,343 & 9,93 & 28 & 2,67 & 40,0 & 2,67 & 15,27 \\
\hline Brosimum discolor Schott & 1,075 & 4,56 & 48 & 4,58 & 62,5 & 4,17 & 13,30 \\
\hline Parkia pendula (Willd.) Benth. ex Walp. & 1,856 & 7,87 & 16 & 1,53 & 27,5 & 1,83 & 11,23 \\
\hline $\begin{array}{l}\text { Schefflera morototoni (Aubl.) Maguire, Steyerm. } \\
\text { \& Frodin }\end{array}$ & 0,862 & 3,65 & 30 & 2,86 & 37,5 & 2,50 & 9,01 \\
\hline Eschweilera ovata (Cambess.) Miers & 0,430 & 1,82 & 32 & 3,05 & 50,0 & 3,33 & 8,21 \\
\hline Cecropia palmata Willd. & 0,657 & 2,78 & 33 & 3,15 & 25,0 & 1,67 & 7,60 \\
\hline Quiina sp. & 1,371 & 5,81 & 6 & 0,57 & 15,0 & 1,00 & 7,38 \\
\hline Nectandra cuspidata (Nees et. Mart.) Nees & 0,454 & 1,93 & 24 & 2,29 & 45,0 & 3,00 & 7,21 \\
\hline
\end{tabular}


TABELA 1: Continuação ...

TABLE 1: Continued ...

\begin{tabular}{|c|c|c|c|c|c|c|c|}
\hline Nome científico & $\begin{array}{l}\text { DoA } \\
(\mathrm{m} 2)\end{array}$ & \begin{tabular}{r|r|} 
DoR \\
$(\%)$
\end{tabular} & \begin{tabular}{c|} 
DA \\
Ind./ha)
\end{tabular} & \begin{tabular}{r|r|}
$\mathrm{DR}$ \\
$(\%)$ \\
\end{tabular} & \begin{tabular}{l|l|} 
FA \\
$(\%)$
\end{tabular} & $\begin{array}{r}\text { FR } \\
(\%) \\
\end{array}$ & \\
\hline Pouteria grandiflora (A. DC.) Baehni & 0,268 & 1,14 & 27 & 2,57 & 35,0 & 2,33 & 6,04 \\
\hline Cupania racemosa (Vell.) Radlk. & 0,178 & 0,76 & 25 & 2,38 & 40,0 & 2,67 & 5,8 \\
\hline Erythroxylum squamatum Sw. & 0,161 & 0,68 & 25 & 2,38 & 37,5 & 2,50 & 5,5 \\
\hline Ocotea gardneri (Meisn.) Mez & 0,360 & 1,53 & 21 & 2,00 & 25,0 & 1,67 & \\
\hline Inga thibaudiana DC. & 0,196 & 0,83 & 18 & 1,72 & 30,0 & 2,00 & \\
\hline Cedrela sp. & 0,180 & 0,76 & 17 & 1,62 & 30,0 & 2,00 & \\
\hline Copaifera langsdorffii Desf. & 0,360 & 1,53 & 14 & 1,34 & 22,5 & 1,50 & \\
\hline Ocotea opifera Mart. & 0,233 & 0,99 & 15 & 1,43 & 22,5 & 1,50 & \\
\hline Pterocarpus violaceus Vogel & 0,313 & 1,33 & 12 & 1,14 & 17,5 & 1,17 & 3,6 \\
\hline Protium heptaphyllum (Aubl.) Marchand & 0,095 & 0,40 & 15 & 1,43 & 25,0 & 1,67 & \\
\hline Stryphnodendron pulcherrimum (Willd.) Hochr. & 0,307 & 1,30 & 10 & 0,95 & 17,5 & 1,17 & 3,4 \\
\hline Eriotheca gracilipes (K. Schum.) A. Robyns & 0,159 & 0,67 & 11 & 1,05 & 22,5 & 1,50 & 3,22 \\
\hline Licania rigida Benth. & 0,298 & 1,26 & 11 & 1,05 & 12,5 & 0,83 & 3,1 \\
\hline Mabea occidentalis Benth & 0,257 & 1,09 & 10 & 0,95 & 15,0 & 1,00 & 3,0 \\
\hline Ouratea hexasperma (A. St.-Hil.) Baill. & 0,111 & 0,47 & 13 & 1,24 & 20,0 & 1,33 & 3,0 \\
\hline Chrysophyllum splendens Spreng. & 0,248 & 1,05 & 11 & 1,05 & 12,5 & 0,83 & 2,9 \\
\hline Macrosamanea pedicellaris (DC.) Kleinhoonte & 0,342 & 1,45 & 6 & 0,57 & 12,5 & 0,83 & 2,85 \\
\hline Miconia hypoleuca (Benth.) Triana & 0,057 & 0,24 & 9 & 0,86 & 22,5 & 1,50 & 2,60 \\
\hline Cupania revoluta Rolfe & 0,040 & 0,17 & 10 & 0,95 & 20,0 & 1,33 & 2,45 \\
\hline Protium giganteum Engl. & 0,046 & 0,20 & 9 & 0,86 & 20,0 & 1,33 & 2,3 \\
\hline Miconia albicans (Sw.) Triana & 0,048 & 0,20 & 10 & 0,95 & 17,5 & 1,17 & 2,3 \\
\hline Ocotea glomerata Benth. \& Hook. & 0,111 & 0,47 & 8 & 0,76 & 15,0 & 1,00 & 2,2 \\
\hline Tabebuia impetiginosa (Mart. ex DC.) Standl. & 0,110 & 0,47 & 8 & 0,76 & 15,0 & 1,00 & 2,2 \\
\hline Sloanea obtusifolia (Moric.) K. Schum. & 0,147 & 0,62 & 6 & 0,57 & 15,0 & 1,00 & 2,2 \\
\hline $\begin{array}{l}\text { Allophylus edulis (A. St.-Hil., Cambess. \& A. } \\
\text { Juss.) Radlk. }\end{array}$ & 0,002 & 0,01 & 1 & 0,10 & 2,5 & 0,17 & \\
\hline Hyeronima alchorneoides Allemão & 0,002 & 0,01 & 1 & 0,10 & 2,5 & 0,17 & 0,27 \\
\hline Citharexylum pernambucense Mold. & 0,239 & 1,01 & 8 & 0,76 & 5,0 & 0,33 & 2,1 \\
\hline Trichilia silvatica C. DC. & 0,052 & 0,22 & 9 & 0,86 & 15,0 & 1,00 & 2,0 \\
\hline Miconia prasina $(\mathrm{Sw}$.$) DC.$ & 0,064 & 0,27 & 8 & 0,76 & 15,0 & 1,00 & 2,0 \\
\hline Matayba cf. elaeagnoides Radlk. & 0,236 & 1,00 & 7 & 0,67 & 5,0 & 0,33 & 2,0 \\
\hline Guapira opposita (Vell.) Reitz & 0,110 & 0,47 & 6 & 0,57 & 12,5 & 0,83 & 1,8 \\
\hline Machaerium aculeatum (Vell.) Stelifeld. & 0,243 & 1,03 & 5 & 0,48 & 5,0 & 0,33 & $1, \varepsilon$ \\
\hline Virola gardneri (A. DC.) Warb. & 0,177 & 0,75 & 4 & 0,38 & 10,0 & 0,67 & 1,8 \\
\hline Himatanthus phagedaenicus (Mart.) Woodson & 0,081 & 0,34 & 8 & 0,76 & 10,0 & 0,67 & 1,7 \\
\hline Mimosa sp. & 0,124 & 0,53 & 6 & 0,57 & 10,0 & 0,67 & 1,7 \\
\hline Rheedia gardneriana Planch. \& Triana & 0,073 & 0,31 & 5 & 0,48 & 12,5 & 0,83 & 1,6 \\
\hline Simarouba amara Aubl. & 0,158 & 0,67 & 4 & 0,38 & 7,5 & 0,50 & 1,5 \\
\hline Myrcia rostrata DC. & 0,054 & 0,23 & 5 & 0,48 & 12,5 & 0,83 & 1,5 \\
\hline Aspidosperma discolor A. DC. & 0,193 & 0,82 & 4 & 0,38 & 5,0 & 0,33 & 1,5 \\
\hline Xylopia frutescens Aubl. & 0,046 & 0,20 & 6 & 0,57 & 10,0 & 0,67 & 1,4 \\
\hline Miconia ligustroides (DC.) Naudin & 0,017 & 0,07 & 5 & 0,48 & 12,5 & 0,83 & 1,3 \\
\hline Inga capitata Desv. & 0,034 & 0,14 & 6 & 0,57 & 10,0 & 0,67 & 1,3 \\
\hline Zanthoxylum petiolare A. St. Hil. & 0,236 & 1,00 & 2 & 0,19 & 2,5 & 0,17 & 1,36 \\
\hline
\end{tabular}


TABELA 1: Continuação ...

TABLE 1: Continued ...

\begin{tabular}{|c|c|c|c|c|c|c|c|}
\hline Nome científico & $\begin{array}{l}\text { DoA } \\
\text { (m2) }\end{array}$ & \begin{tabular}{r|} 
DoR \\
$(\%)$
\end{tabular} & $\begin{array}{c}\text { DA } \\
\text { Ind./ha) }\end{array}$ & $\begin{array}{l}\text { DR } \\
(\%) \\
\end{array}$ & $\begin{array}{r}\text { FA } \\
(\%)\end{array}$ & $\begin{array}{r}\text { FR } \\
(\%) \\
\end{array}$ & VI \\
\hline$\overline{\text { Byrsonima sericea DC. }}$ & 0,028 & 0,12 & 5 & 0,48 & 10,0 & 0,67 & $\overline{1,26}$ \\
\hline Bowdichia virgilioides Kunth & 0,173 & 0,74 & 2 & 0,19 & 5,0 & 0,33 & 1,26 \\
\hline Sorocea hilarii Gaudich. & 0,042 & 0,18 & 4 & 0,38 & 10,0 & 0,67 & 1,22 \\
\hline Amaioua guianensis Aubl. & 0,080 & 0,34 & 4 & 0,38 & 7,5 & 0,50 & 1,22 \\
\hline Hymenaea courbaril L. & 0,129 & 0,55 & 3 & 0,29 & 5,0 & 0,33 & 1,16 \\
\hline Tapirira sp. & 0,048 & 0,20 & 6 & 0,57 & 5,0 & 0,33 & 1,11 \\
\hline Guatteria pogonopus Mart. & 0,048 & 0,20 & 4 & 0,38 & 7,5 & 0,50 & 1,08 \\
\hline Pouteria caimito (Ruiz \& Pav.) Radlk. & 0,063 & 0,27 & 3 & 0,29 & 7,5 & 0,50 & 1,05 \\
\hline Indeterminada 01 & 0,052 & 0,22 & 3 & 0,29 & 7,5 & 0,50 & 1,01 \\
\hline Annona glabra L. & 0,027 & 0,12 & 4 & 0,38 & 7,5 & 0,50 & 1,00 \\
\hline Casearia arborea (L. C. Rich.) Urb. & 0,020 & 0,08 & 4 & 0,38 & 7,5 & 0,50 & 0,97 \\
\hline Pouteria scytalophora Eyma & 0,015 & 0,07 & 3 & 0,29 & 7,5 & 0,50 & 0,85 \\
\hline Casearia cf. commersoniana Cambess & 0,013 & 0,06 & 3 & 0,29 & 7,5 & 0,50 & 0,84 \\
\hline Eschweilera apiculata (Miers) A.C. Sm. & 0,012 & 0,05 & 3 & 0,29 & 7,5 & 0,50 & 0,84 \\
\hline Artocarpus heterophyllus Lam. & 0,031 & 0,13 & 3 & 0,29 & 5,0 & 0,33 & 0,75 \\
\hline Myrciaria tenella Berg. & 0,022 & 0,09 & 3 & 0,29 & 5,0 & 0,33 & 0,71 \\
\hline Cordia nodosa Lam. & 0,023 & 0,10 & 2 & 0,19 & 5,0 & 0,33 & 0,62 \\
\hline Guarea guidonia (L.) Sleumer & 0,007 & 0,03 & 2 & 0,19 & 5,0 & 0,33 & 0,56 \\
\hline Siparuna guianensis Aubl. & 0,007 & 0,03 & 2 & 0,19 & 5,0 & 0,33 & 0,55 \\
\hline Inga laurina (Sw.) Willd. & 0,007 & 0,03 & 2 & 0,19 & 5,0 & 0,33 & 0,55 \\
\hline Casearia sylvestris $\mathrm{Sw}$. & 0,006 & 0,02 & 2 & 0,19 & 5,0 & 0,33 & 0,55 \\
\hline Cecropia sp. & 0,037 & 0,16 & 2 & 0,19 & 2,5 & 0,17 & 0,52 \\
\hline Campomanesia xanthocarpa Berg. & 0,028 & 0,12 & 2 & 0,19 & 2,5 & 0,17 & 0,48 \\
\hline Vismia guianensis (Aubl.) Pers. & 0,012 & 0,05 & 2 & 0,19 & 2,5 & 0,17 & 0,41 \\
\hline Guapira nitida (Schmidt) Lundell & 0,007 & 0,03 & 2 & 0,19 & 2,5 & 0,17 & 0,39 \\
\hline Apeiba tibourbou Aubl. & 0,027 & 0,11 & 1 & 0,10 & 2,5 & 0,17 & 0,38 \\
\hline Schinus terebinthifolius Raddi & 0,022 & 0,09 & 1 & 0,10 & 2,5 & 0,17 & 0,35 \\
\hline Inga edulis (Vell.) Mart. & 0,013 & 0,05 & 1 & 0,10 & 2,5 & 0,17 & 0,32 \\
\hline Casearia javitensis Kunth & 0,011 & 0,05 & 1 & 0,10 & 2,5 & 0,17 & 0,31 \\
\hline Inga fagifolia G. Don. & 0,011 & 0,05 & 1 & 0,10 & 2,5 & 0,17 & 0,31 \\
\hline Trichilia lepidota Mart. & 0,009 & 0,04 & 1 & 0,10 & 2,5 & 0,17 & 0,30 \\
\hline Ocotea sp. & 0,004 & 0,02 & 1 & 0,10 & 2,5 & 0,17 & 0,28 \\
\hline Guatteria cf. schlechtendaliana Mart. & 0,003 & 0,01 & 1 & 0,10 & 2,5 & 0,17 & 0,28 \\
\hline Annona sp. & 0,003 & 0,01 & 1 & 0,10 & 2,5 & 0,17 & 0,27 \\
\hline Total geral & 23,597 & 100,00 & 1049 & 100,00 & 1500 & 100,00 & 300,00 \\
\hline
\end{tabular}

Em que: $\mathrm{VI}$ = valor de importância; $\mathrm{FA}=$ freqüência absoluta; $\mathrm{DA}=$ densidade absoluta; DoA = dominância absoluta; $\mathrm{FR}=$ freqüência relativa; $\mathrm{DR}=$ densidade relativa; $\mathrm{DoR}=$ dominância relativa.

\section{CONCLUSÕES}

O fragmento estudado apresenta alta riqueza florística, quando comparado com a maioria dos estudos em Floresta Ombrófila Densas de Pernambuco;

A distribuição diamétrica observada indica que o fragmento estudado se encontra em estágio de sucessão secundária. 


\section{REFERÊNCIAS BIBLIOGRÁFICAS}

ASSMANN, E. The principles of forest yield: studies in the organic production, structure, increment and yield of forest stands. Braunschweig: Pergamon Press, 1970.506 p.

BORÉM, R.A.T.; OLIVEIRA-FILHO, A. T. Fitossociologia do estrato arbóreo em uma toposeqüência alternada de Floresta Ombrófila Densa, no município de Silva Jardim-RJ, Brasil. Revista Árvore, Viçosa, MG, v. 26, n. 6, p.727742, 2002.

BORÉM, R.A.T.; RAMOS, D.P. Estrutura fitossociológica da comunidade arbórea de uma toposeqüência pouco alterada de uma área de floresta atlântica, no município de Silva Jardim-RJ, Brasil. Revista Árvore, Viçosa, MG, v. 25, n. 1, p.131-140, 2001.

CONDEPE. Catende. Recife: CONDEPE, 1987. 62p. (Monografias Municipais, 27).

CRONQUIST, A. The evolution and classification of flowering plants. $2^{\text {a }}$ ed. The New York Botanical Garden: New York, 1988. 555p.

FEITOSA, A. A. N. Diversidade de espécies arbóreas associada ao solo e toposseqüencia de fragmento de Mata Atlântica de Pernambuco. Recife: UFRPE, 2004. 102p. Dissertação (Mestrado em Ciência do Solo) - Universidade Federal Rural de Pernambuco, 2004.

FERREIRA, R.L.C, VALE, A.B. do. Subsídios básicos para o manejo florestal da caatinga. Revista do Instituto Florestal, São Paulo v. 4, n. único, parte 2, p. 368-375, 1992.

GENTRY, A. H. Diversity and floristic composition of neotropical dry Forest. In: BULLOCK, S. H.; MOONEY, H. A.; MEDINA, E. (Ed) Seasonally dry forest. Cambridge: Cambridge University Press, 1995. p. 146-194.

GUEDES, L. S. A vegetação fanerogâmica da reserva ecológica de Dois Irmãos, Recife-PE. In: Isabel Cristina Machado, Adriana Valentina Lopes \& Kátia Cavalcanti Porto (Organizadores) Reserva Ecológica de Dois Irmãos: estudos em um remanescente de Mata Atlântica em área urbana (recife/Pernambuco/ Brasil) SECTMA, Editora: Universitária, da UFRPE, 1998. 157-172p.

JOLY, C. A.; LEITÃO-FILHO, H. F.; SILVA, S. M. O patrimônio florístico. In: CECCHI, J.C.; SOARES, M.S.M. (Coord.). Mata Atlântica/Atlantic Rain Forest. São Paulo: Fundação SOS Mata Atlântica, 1991. p. 95-125.

LEITÃO FILHO, H. F. Considerações sobre a florística de florestas tropicais e subtropicais do Brasil. IPEF, Piracicaba, n.45, p.41-46, 1987.

LINS-E-SILVA, A. C. B.; RODAL, M. J. N. Tree community structure in an urban atlantic forest remnant in Pernambuco, Brazil. Memoir of New York Botanical Garden, New York, 2007. (no prelo).

LORENZI, H. Árvores brasileiras: manual de identificação e cultivo de plantas arbóreas nativas do Brasil. Nova Odessa: Plantarum, 1992. 352 p.

MACHADO, E.L.M.; OLIVEIRA-FILHO, A.T.; CARVALHO, W.A.C. et al. Análise comparativa da estrutura e flora do compartimento arbóreo-arbustivo de um remanescente florestal na fazenda Beira Lago, Lavras, MG. Revista Árvore, Viçosa, v.28, n.4, p.499-516, 2004.

MAGURRAN, A.E. Ecological diversity and its measurement. Princeton University, New Jersey, 1988.

MARTINS, F. R. Estrutura de uma floresta mesófila. Campinas: UNICAMP, 1991. 245 p.

MEYER, H. A. Structure, growth, and drain in balanced uneven-aged forests. Journal of Forestry, Washington, n. 52, v. 2, p. $85-92,1952$.

MUELLER-DOMBOIS, D.; ELLENBERG, H. Aims and methods of vegetation Ecology. New York: John Wiley \& Sons, $1974.547 \mathrm{p}$.

OLIVEIRA, Z. L.; SANTOS JÚNIOR, R.C.B.; FELICIANO, A.L.P. et al. Levantamento florístico e fitossociológico de um trecho de Mata Atlântica na estação florestal experimental de Nísia floresta - RN. Brasil Florestal, Brasília, DF, v. 71, p. 22-29, 2001.

SAEG - SISTEMA PARA ANÁLISE ESTATÍSTICA E GENÉTICA. Manual de uso. Viçosa-MG: UFV-Funarbe, 1997. 150p.

SILVA JÚNIOR, J. F. Estudo fitossociológico em um remanescente de floresta atlântica visando dinâmica de espécies florestais arbóreas no município do Cabo de Santo Agostinho, PE. Recife: UFRPE, 2004. 82 p. Dissertação (Mestrado em Ciências Florestais) - Universidade Federal Rural de Pernambuco, 2004.

SILVA JÚNIOR, M. C. ; SILVA, A. F. . Distribuição dos diâmetros dos troncos das espécies mais importantes do 
Cerrado na Estação Florestal de Experimentação de Paraopeba, Mina Gerais. Acta Botanica Brasilica, Rio de Janeiro, v. 2, n. 1-2, p.107-126, 1988.

SILVA, A. G. Fisionomia e estrutura da comunidade arbórea na Mata dos Macacos, município de Igarassu-PE. Recife: UFRPE, 2004. 69p. Dissertação (Mestrado em Botânica) - Universidade Federal Rural de Pernambuco, 2004.

SIQUEIRA, D. R.; RODAL, M. J. N.; LINS-E-SILVA, A. C. B.; MELO, A. L. Physiognomy, Structure, and Floristics in an Area of Atlantic Forest in Northeast Brazil. In: Gottsberger, G.; Liede, S. (Org.) Life Forms and Dynamics in Tropical Forests. Berlim-Stuttgart: Gebr. Borntraeger Verlagsbuchhandlung, 2001. p.11-27. (Dissertaciones Botanicae, Band 346).

UNIVERSIDADE FEDERAL DE VIÇOSA - UFV. SAEG - Sistema de análises estatísticas e genéticas. Versão 7.1. Viçosa, MG: UFV, 1997. 150p. (Manual do usuário).

VANINI, A. E.; RODRIGUES, R. R. Caracterização silvigênica em uma formação pioneira com influência marinha no Parque Estadual da Ilha do Cardoso, Cananéia - SP. In: CONGRESSO DE ECOLOGIA DO BRASIL, 6, 2003, Fortaleza. Anais... Fortaleza : Editora da Universidade Federal do Ceará, 2003, v.1, p.180-182 